\title{
1/n Expansion for a Quantum Field Model`
}

\author{
Antti J. Kupiainen \\ Lyman Laboratory of Physics, Harvard University, MA 02138, USA
}

\begin{abstract}
A nonperturbative study of the 1/ $n$ expansion in Euclidean Quantum Field Theory is started. The expansion is shown to be asymptotic to the vacuum energy of the $\left(\phi^{2}\right)_{2}^{2}$ model, for arbitrary coupling constant.
\end{abstract}

\section{Introduction}

The $1 / n$ expansion has recently aroused a great deal of interest among field theorists (for references see [1]). A rigorous, nonperturbative study of this expansion was started in [1], where the lattice nonlinear $\sigma$-model was considered. The techniques of [1] can also be applied to the $\lambda\left(\phi^{2}\right)^{2}$ lattice field theory (in fact this theory is much easier to control; one can prove the estimates uniformly in $\lambda$ as $\lambda \rightarrow \infty)$. However, it is not possible to pass to the continuum limit using these methods. The reason for this is that the random walk expansions which played a central role in the estimates produce a divergence as $\varepsilon$, the lattice spacing, tends to zero. The source for the divergence lies in the fact that we perturbed in the offdiagonal part of $\Delta$ (the lattice Laplacean), which perturbation becomes singular as $\varepsilon \rightarrow 0$.

In the present paper we develop a different method to examine the $1 / n$ expansion for the two dimensional (continuum) $\left(\phi^{2}\right)^{2}$ quantum field theory. In particular we will show that the expansion is asymptotic to the pressure (vacuum energy) for arbitrary large coupling constant. The idea is to derive a closed form expression for the remainder in terms of Schwinger functions of suitable type. These can then be bounded using chessboard estimates and Hölder's inequality. We present two ways to generate the expansion with a remainder, one using a "dual" representation of the model in terms of a complex nonlocal measure, the other using the $\phi$-representation.

The techniques of this paper can be used to derive the expansion for Schwinger functions with the remainder given again in terms of Schwinger functions. The existing cluster expansions can be applied to construct these for small coupling $\lambda$;

* Supported in part by the National Science Foundation under Grant No. PHY 79-16812 
for large $\lambda$ they don't converge because of large pressure contributions. However, the $1 / n$ expansion predicts that the theory is close to gaussian for any $\lambda$ as $n$ becomes large enough. To construct the theory for arbitrary $\lambda$, and thus also to get the expansion for correlations and mass gap, one needs a new cluster expansion which explicitly cancels the diverging pressure factors. This will be discussed in a forthcoming publication.

The contents of the paper are as follows. In Sect. 2 we briefly recall some facts about the model and state the main result. In Sect. 3 the expansion is generated in the $\phi$-representation by successively integrating by parts and obtaining linear integral equations for various correlations, the solution of which constitutes an elementary expansion step. However, the general remainder is quite inexplicit when derived in this fashion and, for example, we only get an $e^{\alpha \lambda} n^{-1}$ type bound for the first order remainder. In Sect. 4 we introduce the dual representation, generate the expansion there and prove the main result.

\section{Description of the Model and Main Results}

Let $\Lambda$ be the (flat) torus obtained from $[-L, L]^{2}, L \in \mathbb{N}$, by identifying opposite sides, and $\Delta_{\Lambda}$ the Laplacean on $L^{2}(\Lambda)$. Let $d \mu_{\Lambda}^{(1)}$ be the gaussian measure on $\mathscr{D}^{\prime}(\Lambda)$ with covariance $C_{\Lambda}=\left(-\Delta_{\Lambda}+1\right)^{-1}$ and $d \mu_{\Lambda}(\phi)=\bigotimes_{i=1}^{n} d \mu_{\Lambda}^{(1)}\left(\phi_{i}\right)$ on $\mathscr{D}^{\prime}(\Lambda)^{n}$. The partition function $Z(\Lambda, \lambda, n)$ is

$$
Z(\Lambda, \lambda, n)=\int e^{V(\Lambda, \lambda, \phi)} d \mu_{\Lambda}(\phi),
$$

where

$$
V(\Lambda, \lambda, \phi)=-\frac{\lambda}{4 n} \int_{\Lambda}:(\phi(x) \cdot \phi(x))^{2}: d^{2} x
$$

and : : denotes Wick-ordering with respect to $d \mu_{\Lambda}, \phi \cdot \phi=\sum_{\alpha=1}^{n} \phi^{(\alpha)} \phi^{(\alpha)}$. We will be using the following properties of this model; the proofs are analogous to those in the $n=1$ case, only the differences are indicated below. (For general information on Euclidean field theory see [2].)

\section{A. Conditioning $[3,4]$}

Let $\Lambda=\Lambda_{1} \cup \Lambda_{2}$ where $\Lambda_{i}$ are open and disjoint. Let $C_{\Lambda_{i}}^{N}$ be the covariance on $L^{2}\left(\Lambda_{i}\right)$ with Neumann boundary conditions on $\partial \Lambda_{i}[4]$ and let $V\left(\Lambda_{i}\right)$ have Wick ordering with respect to $d \mu_{\Lambda_{i}}^{N}$, which has covariance $\bigoplus_{j=1}^{n}\left(C_{\Lambda_{i}}^{N}\right)^{(j)}$ where $\left(C_{\Lambda_{i}}^{N}\right)^{(j)}$ are $n$ copies of $C_{A_{i}}^{N}$. Then [4]

$$
\begin{aligned}
\int e^{V(\Lambda)} d \mu_{\Lambda} & \leqq \int e^{V\left(\Lambda_{1}\right)} d \mu_{\Lambda_{1}}^{N} \int e^{V\left(\Lambda_{2}\right)} d \mu_{\Lambda_{2}}^{N}, \\
\int\left|R_{\Lambda}\right|^{q} d \mu_{\Lambda} & \leqq \int\left|R_{\Lambda_{1} \Lambda_{2}}\right|^{q} d \mu_{\Lambda_{1} \Lambda_{2}} \\
R_{\Lambda} & =\prod_{i} \int g_{i}(x): P_{i}(\phi(x)): d x,
\end{aligned}
$$


and $R_{\Lambda_{1} \Lambda_{2}}$ has Wick ordering with respect to $\bigoplus_{i=1}^{n}\left(C_{\Lambda_{1}}^{N} \oplus C_{\Lambda_{2}}^{N}\right)^{(i)}$, the covariance of $d \mu_{\Lambda_{1} \Lambda_{2}}^{N}$ (here $P_{i}$ are polynomials).

\section{B. Semiboundedness}

This states that $V, e^{V} \in L^{p}\left(\mathscr{D}^{\prime}(\Lambda)^{n}, d \mu_{A}\right)$ for all $p<\infty$ and

$$
1 \leqq Z(\Lambda)^{1 /|\Lambda|}=e^{c_{0}\left(\lambda(\log \lambda)^{2}+1\right)},
$$

where $c_{0}$ is independent on $\Lambda$ and $\lambda$ (and $n$ ).

The proof of (4) is exactly as in [2], Chap. V.2, note that for $\phi_{h}(x) \equiv \int h(x-y) \phi(y) d y$ where $h \in C^{\infty}(\Lambda)$

$$
:\left(\phi_{h} \cdot \phi_{h}\right)^{2}:=\left(\phi_{h}^{2}-n b\right)^{2}-4 b \phi_{h}^{2}+2 n b^{2}
$$

and $\quad b(x)=\int \phi_{h}^{2}(x) d \mu_{\Lambda^{\prime}}$. Thus $\inf \left(:\left(\phi_{h}^{2}\right)^{2}:\right)=-2 b^{2}(n+2)$ and hence $\sup V=\lambda\left(\frac{1}{2}+\frac{1}{n}\right) \int b(x)^{2} d^{2} x$. One can now proceed as in [2] to prove (4) for $|\Lambda|=1$ and using (2) for arbitrary $\Lambda$.

\section{Chessboard Estimates [5]}

Let $F_{i}(\phi)$ be a function of $\phi(x)$ localized at $\Delta_{i}$, the unit cube centered at $i \in \mathbb{Z}^{2}$, such that $F$ is invariant under reflections about the lines $x_{\alpha}=i_{\alpha}, \alpha=1,2$. Define the expectation

$$
\langle G\rangle=\left(\int e^{V} d \mu_{\Lambda}\right)^{-1} \int G e^{V} d \mu_{\Lambda} .
$$

Then

$$
\left|\left\langle\prod_{\Delta_{i} \subset \Lambda} F_{i}(\phi)\right\rangle\right| \leqq \prod_{\Delta_{i} \subset \Lambda}\left|\left\langle\prod_{\Delta_{j} \subset \Lambda} \tau_{j} F_{i}(\phi)\right\rangle\right|^{1 /|\Lambda|},
$$

where $\tau_{j} F_{i}$ is the translate of $F_{i}$ to the square $\Delta_{i+j}$ (addition modulo $\Lambda$ ).

\section{Integration by Parts [6]}

The integration by parts formula we use is

$$
\begin{aligned}
& \left\langle: \phi^{(\alpha)}(x)^{k}: F(\phi)\right\rangle \\
& \quad=\int d y C(x-y)\left\langle: \phi^{(\alpha)}(x)^{k-1}:\left(\frac{\delta}{\delta \phi^{(\alpha)}(y)}+\frac{\delta V}{\delta \phi^{(\alpha)}(y)}\right) F\right\rangle .
\end{aligned}
$$

Using (D) and (C) one can prove the local regularity of the correlation functions

$$
\left\langle\prod_{i=1}^{k}:\left(\phi^{\left(\alpha_{i}\right)}\left(x_{i}\right)\right)^{n_{i}}:\right\rangle \equiv F\left(x_{1}, \ldots, x_{k}: \alpha, n\right)
$$

i.e. that $F \in L_{\log }^{p}\left(\Lambda^{k}\right)$ for $p<\infty$ and $\|F\|_{L^{p}\left(A_{1} \times A_{2} \ldots \times A_{k}\right)}$ is uniformly bounded in $|\Lambda|$. In other words, $F$ has the same local singularities as in the free $(\lambda=0)$ theory. (See e.g. [7], who use weaker $\phi$-bounds.) 
Fig. 1

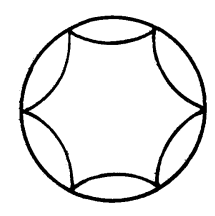

Fig. 2

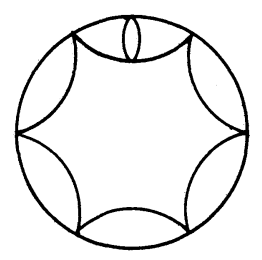

Fig. 3

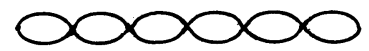

Since one easily gets

$$
\left|\int\left(e^{(1+\varepsilon) V}-e^{V}-\varepsilon V e^{V}\right) d \mu_{\Lambda}\right| \leqq \frac{\varepsilon^{2}}{2}\|V\|_{4}^{2} \cdot \sup _{1 \leqq t \leqq 1+\varepsilon}\left\|e^{t V}\right\|_{2},
$$

(B) implies that $Z(\Lambda, \lambda)$ is differentiable in $\lambda$.

Finally, using conditioning one can prove that the infinite volume pressure (vacuum energy)

$$
p(\lambda)=\lim _{\Lambda \rightarrow \mathbb{R}^{2}} p(\Lambda, \lambda) \equiv \lim _{\Lambda \rightarrow \mathbb{R}^{2}} \log \left(Z(\Lambda, \lambda)^{1 /|\Lambda|}\right)
$$

exists [4].

The perturbation expansion in powers of $\lambda$ for $p$ is easily shown to be asymptotic since it can be generated by integration by parts and the remainder can be bounded using chessboard estimates. Namely, the remainder consists of terms of the form

$$
\int d x_{1} \ldots d x_{k} F\left(x_{1}, \ldots, x_{k} ; \alpha, n_{i}\right) \prod C\left(z_{i}-z_{j}\right),
$$

where $z$ 's are $x$ 's and one fixed point (e.g. the origin) and $C$ 's form a connected tree. One now localizes $C$ 's and uses chessboard estimates (see Sect. 3 for similar computations).

The (formal) $1 / n$ expansion is obtained by formally resuming this asymptotic perturbation expansion. In the graphical representation of the ordinary perturbation expansion, each line carries an index $i$ which is summed from 1 to $n$ and each vertex has a factor $\delta_{i_{1} i_{2}} \delta_{i_{3} i_{4}}$. Thus all graphs of Fig. 1 are $O\left(n^{0}\right)$, whereas those of Fig. 2 are $O\left(n^{-1}\right)$. Such graphs can be formally summed, e.g. the sum of graphs of Fig. 3 is $\frac{\lambda^{2}}{n}(\hat{C} * \hat{C})(1+\lambda \hat{C} * \hat{C})^{-1}$ (here ^ denotes Fourier transform and * 
convolution). The leading term in $1 / n$ for $p$ is the sum of the graphs in Fig. 2. (It should be noted that these sums only converge for small $\lambda$, but the "sum" has a well-defined meaning for large $\lambda$ too; the situation is as that of $(1+\lambda x)^{-1}=\sum_{n=0}^{\infty}(-\lambda x)^{n}$. We note that a systematic formal way to derive the expansion is to introduce a "dual" field $a(x)$ as in the $\sigma$-model [1] and then "integrate out" the $\phi$-fields. We will return to this approach in the fourth section.

We now state the main result, whose proof will be given in Sect. 4 .

Theorem 1. Let $p_{j}$ be the coefficients of the $1 / n$ expansion for the vacuum energy $p$. Then there exist constants $\alpha, \beta>0$, such that for all $m, n \geqq 2 m$ and $\lambda \geqq 2$

$$
p=\sum_{j=0}^{m-1} p_{j} n^{-j}+R_{m},
$$

where

$$
R_{m} \leqq(3 m) ! \lambda^{4} \alpha^{m}\left(\frac{\lambda^{\beta}}{n}\right)^{m}
$$

Remark 1. $\beta$ can be chosen arbitrarily close to 10 , see (53). Also for small $m$ the bound for $\beta$ will be better (see Sect. 3).

Remark 2. Since we are interested in large $\lambda, \lambda \geqq 2$ is assumed (the 2 is quite arbitrary).

Remark 3. By a little extra work, one should be able to show that the $(3 m)$ ! can be replaced by $m$ !.

\section{Non-Perturbative Derivation of the Expansion}

In this section we will generate the $1 / n$ expansion for the pressure by using integration by parts and solving a simple integral equation. Before describing the general case, let us first consider the lowest order. By translation invariance we get (suppressing the $\Lambda$-dependence of $p$ )

$$
\frac{d p}{d \lambda}=-\frac{1}{4 n}\left\langle\int_{\Delta}:\left(\phi^{2}\right)^{2}(x): d x\right\rangle,
$$

where $\Delta$ is a unit square. Thus integrating by parts

$$
\begin{aligned}
\frac{d p}{d \lambda}= & \frac{\lambda}{4 n} \int_{\Delta} d x \int d z C(x-z)\left\langle: \phi^{(1)} \phi^{2}(x):: \phi^{(1)} \phi^{2}(z):\right\rangle \\
= & \frac{\lambda}{4 n}\left(1+\frac{2}{n}\right) \int_{\Delta} d x \int d z C(x-z)^{2}\left\langle: \phi^{2}(x):: \phi^{2}(z):\right\rangle \\
& -\frac{\lambda^{2}}{4 n^{2}} \int_{\Delta} d x \int d z_{1} d z_{2} C\left(x-z_{1}\right) C\left(x-z_{2}\right) \\
& \cdot\left\langle: \phi^{2}(x):: \phi^{(1)} \phi^{2}\left(z_{1}\right):: \phi^{(1)} \phi^{2}\left(z_{2}\right):\right\rangle,
\end{aligned}
$$


where we used in the second step $O(n)$ invariance of $V$ and $d \mu_{A}$. Note that perturbatively the second term is $O\left(n^{-1}\right)$ whereas the first is $O\left(n^{0}\right)$. Also, the first term generates among other things the graphs of Fig. 1. Thus let us consider

$$
G(x-z) \equiv\left\langle: \phi^{2}(x):: \phi^{2}(z):\right\rangle
$$

and denote

$$
: \phi^{(\alpha)} \phi^{2}(x): \equiv v_{\alpha}(x) \quad \text { and } \quad: \phi^{2}(x): \equiv q(x) .
$$

We integrate by parts twice

$$
\begin{aligned}
G(x-z)= & 2 n C(x-z)^{2}-2 \lambda \int d y C(x-y) C(x-z) \\
& \cdot\left\langle v_{1}(y) \phi^{(1)}(z)\right\rangle-\lambda \int d y C(x-y)^{2} G(y-z) \\
& +\frac{\lambda^{2}}{n} \int d y_{1} d y_{2} C\left(x-y_{1}\right) C\left(x-y_{2}\right)\left\langle v_{1}\left(y_{1}\right) v_{1}\left(y_{2}\right) q(z)\right\rangle .
\end{aligned}
$$

(7) and (8) thus lead to the equations

$$
\begin{aligned}
& \frac{d p}{d \lambda}=\frac{(n+2) \lambda}{4 n^{2}} \int_{\Delta} d x \int d z C(x-z)^{2} G(x-z)+R, \\
& G(x-z)=2 n C(x-z)^{2}-\lambda \int d y C(x-y)^{2} G(y-z)+Q(x-z),
\end{aligned}
$$

where

$$
\begin{aligned}
R & =-\frac{\lambda^{2}}{4 n^{2}}\left\langle q(0) v_{1}(C) v_{1}(C)\right\rangle, \\
Q(x) & =-2 \lambda C(x)\left\langle v_{1}(C) \phi^{(1)}(x)\right\rangle+\frac{\lambda^{2}}{n}\left\langle\left(v_{1}(C)\right)^{2} q(x)\right\rangle .
\end{aligned}
$$

Since $C$ is the kernel of a positive operator and $C(x) \in L^{p}(\Lambda)$ for all $p<\infty$, $C(x-z)^{2}$ is the kernel of a positive operator $C^{(2)}$ and we can thus define $K=\left[1+\lambda C^{(2)}\right]^{-1}$ for $\lambda \geqq 0$. In $p$-space $K$ is just the multiplication operator $\hat{K}(p)=[1+\lambda(\hat{C} * \hat{C})(p)]^{-1}$ where $*$ denotes convolution. We can formally solve (10)

$$
G=2 n K C^{(2)}+K Q .
$$

To give (13) a well defined meaning, we need the following Lemma:

Lemma 2. The $x$ space kernel of $K$ can be written as

$$
K(x-y)=\delta(x-y)-\lambda L(x-y),
$$

where $L \in L^{p}(\Lambda)$ for all $p<\infty$, uniformly in $|\Lambda|$. Moreover, for all $\varepsilon>0, p \in(1, \infty)$

$$
\|L\|_{L^{p}\left(\Delta_{i} \times \Delta_{j}\right)} \leqq b(p, \varepsilon) e^{-(1-\varepsilon)|i-j|},
$$

where $b(p, \varepsilon)$ is independent on $\lambda$ and $|\Lambda|$, and $|i-j|$ is the distance on torus.

We will prove Lemma 2 at the end of this section.

By local regularity and Lemma $2 \int K(x-y) Q(y-z) d y$ is well defined and we 
can combine (9), (10), and (13) to obtain

$$
\frac{d p}{d \lambda}=\frac{(n+2)}{4 n}\left[\int L(z) C^{(2)}(z) d z+\frac{1}{n} \int d y L(y) Q(y)\right]+R,
$$

where we also used $C^{(2)} * K=L$.

Note that $\int L C^{(2)}$ is exactly what one calls the sum of the graphs of Fig. 1. We thus have to show, that $R$ and $\frac{1}{n} Q$ are $O\left(\frac{1}{n}\right)$. We define

$$
p_{0}(\lambda)=\frac{1}{4} \int_{0}^{\lambda} d g \int d p g((\hat{C} * \hat{C})(p))^{2}(1+g(C * C)(p))^{-1}
$$

i.e. $p_{0}$ is the leading term to pressure in the expansion. Most of this chapter is devoted to the proof of

Theorem 3. Let $p_{0}(\lambda)$ be defined by (15). Then there exists a constant $\gamma>0$ so that for $\lambda>1$

$$
\left|p(\lambda)-p_{0}(\lambda)\right| \leqq \gamma \lambda^{10} n^{-1}
$$

Remark. As will be evident from what follows, given Eq. (14) it is rather straightforward to bound $R$ and $Q$ so that

$$
\left|p(\lambda)-p_{0}(\lambda)\right| \leqq \gamma_{1} e^{\alpha \lambda(\log \lambda)^{2}} n^{-1 / 2} .
$$

We just use chessboard estimates and semi-boundedness of $e^{V}$. Also, as will be explained below (Theorem 3'), a bound

$$
\left|p(\lambda)-p_{0}(\lambda)\right| \leqq \gamma_{2} e^{\alpha \lambda(\log \lambda)^{2}} n^{-1}
$$

follows by integrating $R$ and $Q$ further by parts and following ideas that lead to (14). The $\lambda^{10}$ factor however needs much harder work; we will obtain it by bounding the general order remainder of the expansion in Sect. 4. Finally we note that perturbation theory suggests $\lambda^{6} n^{-1}$ behavior instead of the $\lambda^{10} n^{-1}$ behavior we get.

We shall now study the terms $R$ and $Q$ of Eqs. (11) and (12). Let us consider e.g. the first term in $Q$

$$
Q_{1}(x)=-2 \lambda C(x)\left\langle v_{1}(C) \phi^{(1)}(x)\right\rangle .
$$

We will estimate $Q_{1}$ using chessboard estimates. Before this we have to integrate by parts and repeat the ideas that lead to (14) for the following reason. Consider e.g. the expectation $\langle q(\Delta)\rangle$. We expect this to be $O\left(n^{0}\right)$. However, a chessboard estimate only gives

$$
|\langle q(\Delta)\rangle| \leqq\left|\left\langle\prod_{\Delta_{i} \subset \Delta} q\left(\Delta_{i}\right)\right\rangle\right|^{1 /|\Lambda|}
$$

which at least in perturbation theory is $O\left(n^{1 / 2}\right)$. [Combined with what follows this leads to (16)]. However we compute [note that $\langle q(\Delta)\rangle=\langle q(0)\rangle]$

$$
\begin{aligned}
\langle q(0)\rangle & =\lambda \int d x C(x)\left\langle\phi^{(1)}(0) v_{1}(x)\right\rangle \\
& =-\lambda\left[\int d x C(x)^{2}\right]\langle q(0)\rangle+\frac{\lambda^{2}}{n^{2}}\left\langle\left(v_{1}(C)\right)^{2}\right\rangle
\end{aligned}
$$


and thus

$$
\langle q(0)\rangle=\frac{\lambda^{2}}{n}\left(1+\lambda \int C(x)^{2} d x\right)^{-1}\left\langle\left[v_{1}(C)\right]^{2}\right\rangle .
$$

Now note, that the expectation on RHS of (19) is $O(n)$ in perturbation theory and this is what we will prove now. Let us first localize $C ; C=\sum C_{i}$ where $C_{i}=C \chi_{i}, \chi_{i}$ being the characteristic function of the unit square $\Delta_{i}$ centered at $i \in \mathbb{Z}^{2}$. We apply chessboard estimates and Hölder's inequality $\left(q\right.$ even and $\left.\langle\cdot\rangle_{0}=\int \cdot d \mu_{\Lambda}, i \neq j\right)$

$$
\begin{aligned}
& \left\langle v_{1}\left(C_{i}\right) v_{1}\left(C_{j}\right)\right\rangle \leqq\left|\left\langle\prod_{k} \tau_{k} v_{1}\left(C_{i}\right)\right\rangle\right|^{1 /|\Lambda|}\left|\left\langle\prod_{k} \tau_{k} v_{1}\left(C_{j}\right)\right\rangle\right|^{1 /|\Lambda|} \\
& \leqq \prod_{\alpha=i, j}\left\langle\left(\prod_{k} \tau_{k} v_{1}\left(C_{\alpha}\right)\right)^{q}\right\rangle 0^{1 / q|\Lambda|}\left\langle e^{r V}\right\rangle_{0}^{1 / r|\Lambda|} \\
& \cdot\left\langle e^{V}\right\rangle_{0}^{-1 /|\Lambda|} .
\end{aligned}
$$

Here $r^{-1}+q^{-1}=1$. Let $\langle\cdot\rangle_{\Delta_{i}}$ be $\int \cdot d \mu_{\Delta_{i}}^{N}$ where $d \mu_{\Delta_{i}}^{N}$ has Neumann data on $\partial \Delta_{i}$ (2.B). Since $\tau_{k} v_{1}\left(C_{\alpha}\right)$ is measurable with respect to the $\sigma$-algebra generated by the fields on $\Delta_{\alpha+k}$ we get, by conditioning to the covariance $\bigoplus_{j} C_{\Delta_{j}}^{N}$,

$$
\left\langle\left(\prod_{k} \tau_{k} v_{1}\left(C_{\alpha}\right)\right)^{q}\right\rangle_{0}^{1 / q|\Lambda|} \leqq\left\langle v_{1}\left(C_{\alpha}\right)^{q}\right\rangle_{\Delta_{\alpha}}^{1 / q}
$$

Thus

$$
\left|\left\langle v_{1}\left(C_{i}\right) v_{1}\left(C_{j}\right)\right\rangle\right| \leqq k_{i j} \exp \left(r^{-1} p(r \lambda)-p(\lambda)\right),
$$

where

$$
k_{i j}=\left\{\begin{array}{lll}
\prod_{\alpha=i, j}\left\langle v_{1}\left(C_{\alpha}\right)^{q}\right\rangle_{\Delta_{\alpha}}^{1 / q} & \text { if } & i \neq j \\
\left\langle v_{1}\left(C_{i}\right)^{2 q}\right\rangle_{\Delta_{i}}^{1 / q} & \text { if } & i=j .
\end{array}\right.
$$

We thus have to estimate the gaussian expectation $\left\langle v_{1}\left(C_{i}\right)^{q}\right\rangle_{\Delta_{\imath}}=\left\langle\left(: \phi^{(1)} \phi^{2}:\left(C_{i}\right)\right)^{q}\right\rangle_{\Delta_{i}}$.

Lemma 4. Let $q$ be even and $n>\alpha q, \alpha \leqq \frac{1}{2}$. Then

$$
\left\langle v_{1}\left(C_{i}\right)^{q}\right\rangle_{\Delta_{i}}^{1 / q} \leqq b_{0} \alpha^{-1 / 2} q n^{1 / 2}\left\|C_{i}\right\|_{L^{4}\left(\Delta_{t}\right)},
$$

where $b_{0}$ is independent on $q, n, \lambda,|\Lambda|$.

The proof of Lemma 4 is given at the end of this section.

We can now estimate $Q_{1}$ of (18):

Lemma 5. $\left|Q_{1}(x)\right| \leqq b_{1} \lambda^{2} q^{2} C(x) \exp \left[r^{-1} p(r \lambda)-p(\lambda)\right]$.

Proof. Integrating by parts

$$
\begin{aligned}
\left\langle v_{1}(y) \phi^{(1)}(x)\right\rangle & =C(x-y)\langle q(y)\rangle-\frac{\lambda}{n} \int d u C(x-u)\left\langle v_{1}(y) v_{1}(u)\right\rangle \\
& =\frac{\lambda^{2}}{n} C(x-y)\left(1+\lambda \int C(x)^{2} d x\right)^{-1}\left\langle v_{1}(C)^{2}\right\rangle-\frac{\lambda}{n}\left\langle v_{1}(y) v_{1}\left(C_{x}\right)\right\rangle,
\end{aligned}
$$


where we used (19) and denoted $C_{x}=C(\cdot-x)$. Thus

$$
\begin{aligned}
Q_{1}(x)= & -\frac{2 \lambda^{3}}{n} C(x)(C * C)(x)\left[1+\lambda \int C(x)^{2} d x\right]^{-1}\left\langle v_{1}(C)^{2}\right\rangle \\
& +\frac{2 \lambda^{2}}{n} C(x)\left\langle v_{1}(C) v_{1}\left(C_{x}\right)\right\rangle .
\end{aligned}
$$

Since $\left\|C_{i}\right\|_{L^{4}\left(\Delta_{i}\right)} \leqq A e^{-|i|}$ we obtain using Lemma 4 and (20)

$$
\left\langle v_{1}\left(C_{x}\right) v_{1}(C)\right\rangle \leqq B n q^{2} \exp \left(r^{-1} p(r \lambda)-p(\lambda)\right) .
$$

The claim follows, since $(C * C)(x)$ is bounded as is $\lambda\left[1+\lambda \int C(x)^{2} d x\right]^{-1}$.

To conclude our analysis of the first order remainder, we will now estimate $R$ and $Q_{2}=Q-Q_{1}$ [see (11), (12), and (18)]. Both of them have the factor

$$
\left\langle v_{1}\left(x_{1}\right) v_{1}\left(x_{2}\right) q\left(x_{3}\right)\right\rangle \text {. }
$$

We want to express (21) as a sum of terms involving even number of $\phi^{2}$ factors ( $v$ 's are included), for which chessboard estimates give the correct $n$ dependence as we noted above. We integrate by parts

$$
\begin{aligned}
\left\langle v_{1}\left(x_{1}\right) v_{1}\left(x_{2}\right) q\left(x_{3}\right)\right\rangle= & \left(1+\frac{2}{n}\right)\left\langle q\left(x_{1}\right) q\left(x_{2}\right) q\left(x_{3}\right)\right\rangle \\
& \cdot C\left(x_{1}-x_{2}\right)+2 C\left(x_{1}-x_{3}\right)\left\langle q\left(x_{1}\right) v_{1}\left(x_{2}\right) \phi^{(1)}\left(x_{2}\right)\right\rangle \\
& -\frac{\lambda}{n}\left\langle v_{1}\left(C_{x_{1}}\right) v_{1}\left(x_{2}\right) q\left(x_{1}\right) q\left(x_{3}\right)\right\rangle .
\end{aligned}
$$

[Recall that $C_{x}=C\left(\cdot-x\right.$.] The last two terms have even number of $\phi^{2}$ factors. We thus leave them as such. We denote in the first term $q\left(x_{2}\right) q\left(x_{3}\right)$ by $P$, and integrate $q\left(x_{1}\right)$ twice by parts :

$$
\begin{aligned}
\left\langle q\left(x_{1}\right) P\right\rangle= & n\left\langle\phi^{(1)}\left(x_{1}\right) \frac{\delta P}{\delta \phi^{(1)}}\left(C_{x_{1}}\right)\right\rangle \\
& -\lambda \int C\left(x_{1}-y\right)^{2}\langle q(y) P\rangle d y-\lambda\left\langle v_{1}\left(C_{x_{1}}\right) \frac{\delta P}{\delta \phi^{(1)}}\left(C_{x_{1}}\right)\right\rangle \\
& +\lambda^{2} n^{-1}\left\langle v_{1}\left(C_{x_{1}}\right) v_{1}\left(C_{x_{1}}\right) P\right\rangle .
\end{aligned}
$$

Let us now solve this linear integral equation

$$
\begin{aligned}
\left\langle q\left(x_{1}\right) P\right\rangle= & \lambda^{2} n^{-1} \int K\left(x_{1}-y\right)\left\langle v_{1}\left(C_{y}\right) v_{1}\left(C_{y}\right) P\right\rangle d y \\
& -\lambda \int d y K\left(x_{1}-y\right)\left\langle v_{1}\left(C_{y}\right) \frac{\delta P}{\delta \phi^{(1)}}\left(C_{y}\right)\right\rangle \\
& +n \int d y K\left(x_{1}-y\right)\left\langle\phi^{(1)}(y) \frac{\delta P}{\delta \phi^{(1)}}\left(C_{y}\right)\right\rangle .
\end{aligned}
$$

Note, that (22) holds for any $O(n)$ invariant $P$. For the case at hand

$$
\frac{\delta P}{\delta \phi^{(1)}}\left(C_{y}\right)=2\left[\phi^{(1)}\left(x_{2}\right) q\left(x_{3}\right) C\left(y-x_{2}\right)+\phi^{(1)}\left(x_{3}\right) q\left(x_{2}\right) C\left(y-x_{3}\right)\right] .
$$


Thus in (22) only the last term has odd number of $\phi^{2}$ factors. We compute

$$
\begin{aligned}
\left\langle\phi^{(1)}(y) \phi^{(1)}\left(x_{2}\right) q\left(x_{3}\right)\right\rangle= & C\left(y-x_{2}\right)\left\langle q\left(x_{3}\right)\right\rangle \\
& +2 C\left(y-x_{3}\right)\left\langle\phi^{(1)}\left(x_{2}\right) \phi^{(1)}\left(x_{3}\right)\right\rangle \\
& -\frac{\lambda}{n}\left\langle v_{1}\left(C_{y}\right) \phi^{(1)}\left(x_{2}\right) q\left(x_{3}\right)\right\rangle .
\end{aligned}
$$

Since $\left\langle q\left(x_{3}\right)\right\rangle$ is given by (19), we have now reduced (21) to a sum of terms including only even number of $\phi^{2}$ factors. These factors are now estimated using chessboard estimates as in the case of $Q_{1}$. Using Hölder's inequality with exponent $q$, each $\phi^{2}$ brings a factor $(q n)^{1 / 2}$ and each $\phi^{(1)} q^{1 / 2}$ as in Lemma 4 . The highest power of $\lambda$ is $\lambda^{2}$ and of $q q^{5 / 2}$. To estimate the kernels, we apply Lemma 2 . We get thus $\left(r^{-1}+q^{-1}=1\right)$.

Lemma 6. (a) $\left|Q_{2}(x)\right| \leqq b_{2} \lambda^{5} q^{5 / 2} \exp \left[r^{-1} p(r \lambda)-p(\lambda)\right]$.

(b) $|R| \leqq b_{3} \lambda^{5} q^{5 / 2} n^{-1} \exp \left[r^{-1} p(r \lambda)-p(\lambda)\right]$.

Lemmas 5 and 6 together with (14) and (15) imply

Proposition 7. $\left|\frac{d p}{d \lambda}-\frac{d p_{0}}{d \lambda}\right| \leqq b n^{-1} \lambda^{6} q^{5 / 2} \exp \left[r^{-1} p(r \lambda)-p(\lambda)\right]$.

Choosing $q=2$ and using (4), we immediately get the result anticipated in the remark following Theorem 3 :

Theorem $3^{\prime}$. Let $p_{0}$ be the leading term in the $1 / n$ expansion for the the pressure $p$. Then

$$
\left|p-p_{0}\right| \leqq A n^{-1} \exp \left[\beta \lambda(\log \lambda)^{2}\right] .
$$

for some $A, \beta$ independent on $\lambda, n$.

To prove Theorem 3 we need an a priori bound for $p-p_{0}$ :

Lemma 8. There exists a $\delta>0$ such that $\left|p(\lambda)-p_{0}(\lambda)\right| \leqq 1$ as $\frac{\lambda^{10}}{n}<\delta$.

Theorem 3 is now an easy consequence of Proposition 7 and Lemma 8 since we can write

$$
\begin{aligned}
r^{-1} p(r \lambda)-p(\lambda)= & r^{-1}\left(p(r \lambda)-p_{0}(r \lambda)\right)+\left(p_{0}(\lambda)-p(\lambda)\right) \\
& +\left(r^{-1} p_{0}(r \lambda)-p_{0}(\lambda)\right) .
\end{aligned}
$$

By Lemma 8 we need to consider only the last term. From an explicit formula for the convolution $C^{(2)}(p)$ [see e.g. [8], formula (46)]

$$
\alpha_{2}\left(p^{2}+1\right)^{-1} \leqq C^{(2)} \leqq \alpha_{1} \frac{\log \left(p^{2}+2\right)}{p^{2}+1} .
$$


Thus, since $\lambda \geqq 2$

$$
\left|\frac{d p_{0}}{d \lambda}\right|=\frac{\lambda}{4} \int\left(C^{(2)}\right)^{2}\left(1+\lambda C^{(2)}\right)^{-1}<\alpha_{3}(\log \lambda)^{2} .
$$

Hence there is $\alpha_{4}>0$ such that $q>\alpha_{4} \lambda(\log \lambda)^{2}$ implies $\left|r^{-1} p_{0}(r \lambda)-p_{0}(\lambda)\right|<1$. This proves Theorem 3 .

We will prove Lemma 8 by estimating the general order remainder and expansion coefficients. Let

$$
p(\lambda)=\sum_{k=0}^{l-1} p_{k} n^{-k}+R_{l}
$$

be the $1 / n$ expansion with remainder. The main estimate of Sect. 4 is

Lemma 9. Let $2 k+2<n$. Then

(a) $\left|p_{k}\right| \leqq(3 k) ! \lambda^{6 k+2} C_{0}^{k}$

(b) $\left|R_{k}\right| \leqq(3 k) ! \lambda^{6 k+2} n^{-k} C_{1}^{k} e^{\beta \lambda(\log \lambda)^{2}}$,

where $C_{0} \leqq C_{1}, \beta$ are constants, independent on $\lambda, n, k$.

Remark. (a) is obviously not optimal.

Assuming Lemma 9 we can now prove Lemma 8. Let $x=\left(\lambda^{6} \frac{C_{1}}{n}\right)^{1 / 3}$. Choosing $3 k=x^{-1}$ we get

$(3 k) ! x^{3 k} \leqq \gamma \exp (-1 / x)$.

Thus for $C_{1} \lambda^{6} n^{-1}<\left(2 \beta \lambda(\log \lambda)^{2}\right)^{-3}$ one obtains

$$
\left|R_{k}\right| \leqq \gamma \lambda^{2} \exp \left(-C_{2}\left(n \lambda^{-6}\right)^{1 / 3}\right) .
$$

For $\lambda^{10} n^{-1}$ small we thus get the claim since

$$
\begin{aligned}
\sum_{l=1}^{k-1} p_{l} n^{-l} & \leqq \lambda^{2} \sum_{l=1}^{k-1}(3 l) !\left(c_{1} \frac{\lambda^{6}}{n}\right)^{l}=\lambda^{2} \sum_{l=1}^{k-1} \frac{(3 l) !}{(3 k)^{3 l}} \\
& \leqq \lambda^{2} \gamma_{1} \sum_{l=1}^{k-1}\left(\frac{l}{k}\right)^{3 l} e^{-3 l}<\frac{\lambda^{2} \gamma_{1}}{k^{3}} \sum_{l=1}^{k-1} l^{3} e^{-3 l} \leqq \gamma_{2} \frac{\lambda^{8}}{n}
\end{aligned}
$$

We will close this section by showing how the expansion and the general remainder $R_{k}$ can be obtained using the ideas we have explained. For this purpose, we consider the expectation

$$
\left\langle q\left(y_{1}\right) q\left(y_{2}\right) P\right\rangle \text {, }
$$

where we assume $P$ is $O(n)$ invariant. Perturbation theory suggests that $q q$ gives rise to an $L$. This is achieved by integration by parts twice and solving the resulting 
integral equation:

$$
\begin{aligned}
\left\langle q\left(y_{1}\right) q\left(y_{2}\right) P\right\rangle=2 & (n+2) L\left(y_{1}-y_{2}\right)\langle P\rangle \\
+ & (n+2) \int d z_{1} d z_{2} K\left(y_{1}-z_{1}\right) C\left(z_{1}-y_{2}\right) C\left(z_{1}-z_{2}\right) \\
& \cdot\left\langle\phi^{(1)}\left(y_{2}\right) \frac{\delta P}{\delta \phi^{(1)}\left(z_{2}\right)}\right\rangle \\
- & \lambda(1+2 / n) \int d z_{1} d z_{2} K\left(y_{1}-z_{1}\right) C\left(z_{1}-y_{2}\right) C\left(z_{1}-z_{2}\right) \\
& \cdot\left\langle\phi^{(1)}\left(y_{2}\right) v_{1}\left(z_{2}\right) P\right\rangle \\
+ & (n+2) \int d z_{1} d z_{2} C\left(z_{1}-z_{2}\right) K\left(y_{1}-z_{1}\right) \\
& \cdot\left\langle\phi^{(1)}\left(z_{1}\right) q\left(y_{2}\right) \frac{\delta P}{\delta \phi^{(1)}\left(z_{2}\right)}\right\rangle .
\end{aligned}
$$

$\left[O(n)\right.$ invariance was used in relations such as $\left\langle:\left(\phi^{(\alpha)}\right)^{2}: P\right\rangle=n^{-1}\left\langle: \phi^{2}: P\right\rangle$.] Let us now specialize to the case $P=\prod_{i=3}^{2 k} q\left(y_{i}\right)$. Then the expectation in the fourth term of (24) is

$$
\begin{aligned}
& 2 \sum_{i=3}^{2 k} \delta\left(z_{2}-y_{i}\right)\left\langle\phi^{(1)}\left(z_{1}\right) \phi^{(1)}\left(y_{i}\right) \prod_{\substack{j \geq 2 \\
j \neq i}} q\left(y_{j}\right)\right\rangle \\
& \quad=2 \sum_{i=3}^{2 k} \delta\left(z_{2}-y_{i}\right)\left[C\left(z_{1}-y_{i}\right) \prod_{\substack{j \geq 2 \\
j \neq i}} q\left(y_{j}\right)\right. \\
& \quad+2 \sum_{k \neq j} C\left(z_{1}-y_{k}\right)\left\langle\phi^{(1)}\left(y_{i}\right) \phi^{(1)}\left(y_{k}\right) \prod_{\substack{j \neq i, k \\
j \leqq 2}} q\left(y_{j}\right)\right\rangle \\
& \left.\quad-\frac{\lambda}{n} \int d z_{3} C\left(z_{1}-z_{3}\right)\left\langle v_{1}\left(z_{3}\right) \phi^{(1)}\left(y_{i}\right) \prod_{\substack{j \neq i \\
j \geqq 2}} q\left(y_{j}\right)\right\rangle\right] .
\end{aligned}
$$

We see, that the first term in (25), when inserted to (24), involves

$$
\int d z_{1} K\left(y_{1}-z_{1}\right) C\left(z_{1}-y_{i}\right)^{2}=L\left(y_{1}-y_{i}\right) \text {. }
$$

We have thus derived

$$
\left\langle\prod_{i=1}^{2 k} q\left(y_{i}\right)\right\rangle=2(n+2) \sum_{j=2}^{2 k} L\left(y_{1}-y_{j}\right)\left\langle\prod_{\substack{i \geq 2 \\ i \neq j}} q\left(y_{i}\right)\right\rangle+T,
$$

where $T$ can be read off from (25) and (24). Note, that in perturbation theory $T$ is $O\left(n^{-1}\right)$ compared to the first term. Recall, that in (22) we derived a similar equation for $\left\langle q_{1}(x) P\right\rangle$. The $1 / n$ expansion is now generated by applying (22) and (26) repeatedly to (7) as follows: A general expectation we will encounter is

$$
E=\left\langle\prod_{i=1}^{p_{1}} v_{\alpha_{i}}\left(x_{i}\right) \prod_{j=1}^{p_{2}} q\left(y_{j}\right) \prod_{k=1}^{p_{3}} \phi^{\left(\beta_{k}\right)}\left(z_{k}\right)\right\rangle \text {. }
$$

We distinguish between two different cases depending whether $p_{1}+p_{2}$ is even or odd. In the even case, the $n$ structure can already be seen in perturbation theory on 
the tree level, in the odd case not necessarily

$$
r=p_{1}+p_{2} \text { is even . }
$$

We integrate $\phi^{\left(\alpha_{i}\right)}\left(x_{i}\right)$ 's and $\phi^{\left(\beta_{k}\right)}\left(z_{k}\right)$ 's by parts. Thus

$$
E=\left(\sum \prod C\right)\left\langle\prod_{j} q\left(x_{i}\right) \prod q\left(y_{j}\right)\right\rangle+E^{\prime} \equiv E_{0}^{\prime}+E^{\prime},
$$

where $E^{\prime}$ denotes all the other resulting terms. Note that perturbatively $E^{\prime}$ is of lower order in $1 / n$. To $E_{0}^{\prime}$ we now apply (26), obtaining a sum of terms with $r-2 q$ factors and a remainder $T_{1}$. We repeat this $\frac{1}{2}(r-2)$ times thus obtaining a sum of terms with no expectation and a remainder $\sum_{i} T_{i}$. Hence

$$
E=E_{0}+E_{1},
$$

where $E_{0}$ is the leading term in $1 / n$ expansion for $E$ and $E_{1}$ is an explicit remainder consisting again of terms of the form of (27)

$$
r=p_{1}+p_{2} \text { is odd }
$$

We proceed as in (A) to get (28). $E^{\prime}$ has now $r$ even and thus by (A)

$$
E^{\prime}=\left(E^{\prime}\right)_{0}+\left(E^{\prime}\right)_{1} \text {. }
$$

To $E_{0}^{\prime}$ we apply (22)

$$
E_{0}^{\prime}=\sum_{i=1}^{3} E^{(i)}
$$

The first two terms have $r$ even and by $(\mathrm{A})$

$$
\left.E^{(i)}=\left(E^{(i)}\right)_{0}+E^{(i)}\right)_{1} \quad i=1,2 .
$$

In $E^{(3)} r$ is replaced by $r-2$. We proceed with $\frac{1}{2}(\mathrm{r}-1)$ steps to arrive to (29) again.

The remainder $E_{1}$ in (29) in general consists of terms of the form of (27) with $r$ odd or even. As we have noted before, to get an effective estimate for such terms $r$ has to be even. Thus our final step is

$r$ is odd for a term in $E_{1}$

We proceed as in (A) to get (28). $E^{\prime}$ has $r$ even. To $E_{0}^{\prime}$ we apply (22) repeatedly until all terms are even.

Note, that we have already followed this procedure when we derived (14) from (7) using (A) and applied (C) to (21).

We can now easily see the structure of $p_{k}$ and $R_{k}$ as obtained using steps (A)-(C). Upon expanding $K=1-\lambda L, p_{k}$ consists of connected graphs with lines $C$ and $L$ and vertices $C C C C$ and $C L C . R_{k}$ involves connected graphs with lines and vertices as in $p_{k}$, but with external legs $v_{\alpha_{i}}\left(x_{i}\right), q\left(y_{j}\right), \phi^{\left(\beta_{k}\right)}\left(z_{k}\right)$. That is, each $v_{\alpha}$ comes in the form $\int C(y-x) v_{\alpha}(x) d x$ etc. These terms can be now estimated using chessboard estimates. However, the number of terms and $(\lambda, n)$-structure is much more transparent if we generate the expansion in a different way, which we shall do in Sect. 4. Before this we prove the Lemmas 2 and 4.

Proof of Lemma 2. We can assume $\Lambda=\mathbb{R}^{2}$ (if not, we use Fourier series instead of integrals below). Now

$$
\hat{L}=-(\hat{C} * \hat{C})(1+\lambda \hat{C} * \hat{C})^{-1}
$$


and

$$
(\hat{C} * \hat{C})(p)=\int d^{2} k\left[(k-p)^{2}+1\right]^{-1}\left[k^{2}+1\right]^{-1} .
$$

(30) defines an analytic continuation of $\hat{C} * \hat{C}$ to the region $D=\left\{p \in \mathbb{C}^{2} \mid\|\operatorname{Imp}\|<1\right\}$. Since for $p=p_{1}+i p_{2}$ we have

$$
\left[(k-\bar{p})^{2}+1\right]=\left(k-p_{1}\right)^{2}+2 i p_{2}\left(k-p_{1}\right)+1-p_{2}^{2},
$$

we see that $\operatorname{Re}(\hat{C} * \hat{C})(p)>0$ in $D$. (Of course $\hat{C} * \hat{C}$ is analytic for $\|\operatorname{Imp}\|<2$, but positivity of the real part is not obvious there.) Hence $\hat{L}$ has an analytic continuation to $D$. We write $\hat{L}=-\hat{C} * \hat{C}+\hat{M}$, where $\hat{M}=-\lambda(\hat{C} * \hat{C})^{2}(1+\hat{C} * \hat{C})^{-1}$ belongs to $L^{1}\left(\mathbb{R}^{2}\right)$, since $\hat{C} * \hat{C}>0$ and $\|\hat{C} * \hat{C}\|_{2} \leqq\|\hat{C}\|_{4 / 3}^{2}$ by Young's inequality. Thus $M$ is continuous and since $(\hat{C} * \hat{C})^{2}=C^{2}$ is $C^{\infty}$ in $\mathbb{R}^{2} \backslash\{0\}, L$ is continuous there. It would be now easy to conclude that $L$ has exponential fall-off. However, to control the $\lambda$-dependence of the bound we have to be more careful. Let $e_{b}(x)=e^{b \cdot x}$. Let $\Delta_{i} \cap \Delta_{j}=\emptyset$. By the mean value theorem, for all $b \in \mathbb{R}^{2}$ we can find $x_{k}(b)$ in $\Delta_{k}$, $k=i, j$, such that

$$
\|L\|_{L^{p}\left(\Delta_{i} \times \Delta_{j}\right)}=e_{-b}\left(x_{i}(b)-x_{j}(b)\right)\left\|L e_{b}\right\|_{L^{p}\left(\Delta_{i} \times \Delta_{j}\right)}
$$

( $L$ is continuous in $\mathbb{R}^{2} \backslash\{0\}$ ). By the Hausdorff-Young inequality

$$
\left\|L e_{b}\right\|_{L^{p}\left(\Delta_{i} \times \Delta_{j}\right)} \leqq\left\|L e_{b}\right\|_{p} \leqq \gamma_{p}\|\hat{L}(\cdot-i b)\|_{q} .
$$

As we saw above $\operatorname{Re}(\hat{C} * \hat{C})(p+i b)>0$ for $\|b\|<1$. Thus we get using Young's inequality

$$
\|\hat{L}(\cdot-i b)\|_{q} \leqq\|(\hat{C} * \hat{C})(\cdot-i b)\|_{q} \leqq\|\hat{C}(\cdot-i b)\|_{r}\|\hat{C}\|_{r},
$$

where $r=\left(1-(2 p)^{-1}\right)^{-1}$. Hence we have shown, that

$$
e_{b}\left(x_{i}(b)-x_{j}(b)\right)\|L\|_{L^{p}\left(\Delta_{i} \times \Delta_{j}\right)} \leqq b_{1}(p, \varepsilon)
$$

for $\|b\| \leqq 1-\varepsilon, b_{1}$ being independent on $\lambda$. The second assertion for $\Delta_{i} \cap \Delta_{j}=\emptyset$ now follows by choosing $-b$ parallel to $(i-j)$ and changing $b_{1}$ a bit. If $\Delta_{i} \cap \Delta_{j} \neq \emptyset$, we choose $b=0$ and proceed as above. The first claim follows from the second, since we showed, that $L$ is continuous in $\mathbb{R}^{2} \backslash\{0\}$ and has a logarithmic singularity at the origin.

Proof of Lemma 4. We expand $\phi^{2}$ :

$$
\begin{aligned}
\left\langle\left(: \phi^{(1)} \phi^{2}:\left(C_{i}\right)\right)^{2}\right\rangle_{\Delta_{i}} \\
\quad=\sum_{\alpha_{1}, \ldots, \alpha_{q}=1}^{n}\left\langle\prod_{j=1}^{q}: \phi^{(1)}\left(\phi^{\left(\alpha_{j}\right)}\right)^{2}:\left(C_{i}\right)\right\rangle_{\Delta_{i}} \\
\quad=\sum_{\sum_{j=1}^{n} q_{j}=q}\left\langle\prod_{j=1}^{n}\left(: \phi^{(1)}\left(\phi^{(j)}\right)^{2}:\left(C_{i}\right)\right)^{q_{j}}\right\rangle_{\Delta_{i}} \\
\quad=\sum_{\sum q_{j}=q}^{j} \int_{\Delta^{q}} d x_{1} \ldots d x_{q}\left\langle\prod_{i=1}^{q_{1}}:\left(\phi^{(1)}\right)^{3}\left(x_{i}\right): \prod_{k=q_{1}}^{q} \phi^{(1)}\left(x_{k}\right)\right\rangle_{\Delta_{i}} \\
\quad \cdot \prod_{l=2}^{n}\left\langle\prod_{j=q_{l-1}+1}^{q_{l}}:\left(\phi^{(l)}\right)^{2}\left(x_{j}\right):\right\rangle_{\Delta_{i}} \prod_{m=1}^{q} C_{i}\left(x_{m}\right) .
\end{aligned}
$$


In the last step we used the fact that $\phi^{(l)}$ 's are independent random variables and $\sum^{\prime}$ means that $q_{j} \neq 1$ for $j \geqq 2$ since $\left\langle: \phi^{2}:\right\rangle_{\Delta_{i}}=0$. We now apply Wick's theorem and get a sum of terms

$$
\int_{\Delta^{q}} d x_{1} \ldots d x_{q} \prod_{j=1}^{q} C\left(x_{j}\right) \prod_{\langle\alpha, \beta\rangle} C_{\Delta_{i}}^{N}\left(x_{\alpha}-x_{\beta}\right),
$$

where same $\alpha$ occurs in three $C_{\Delta_{i}}^{N}\left(x_{\alpha}-x_{\beta}\right)$. Using Hölder's inequality repeatedly, (31) is bounded by

$$
\left\|C_{i}\right\|_{L^{4}\left(\Delta_{i}\right)}^{q}\left\|C_{\Delta_{i}}^{N}\right\|_{L^{4}\left(\Delta_{i} \times \Delta_{i}\right)}^{3 q / 2} .
$$

Thus

$$
\left\langle v_{1}\left(C_{i}\right)^{q}\right\rangle \leqq A B^{q}\left\|C_{i}\right\|_{L^{4}\left(\Delta_{i}\right)}^{q},
$$

where

$$
A=\sum_{i=1}^{\sum_{i=1}^{n} q_{i}=q}{ }^{\prime}\left(\frac{q}{2}+q_{1}\right) ! \prod_{j=2}^{n} q_{j} !
$$

since $\left\langle\phi^{2 n}\right\rangle$ has $\frac{2 n !}{2^{n} n !}<e^{O(n)} n !$ terms. To estimate $A$, let $q_{j_{k}} \neq 0$ for $k=1, \ldots, m, j_{k} \geqq 2$ and other $q_{j}=0, j \geqq 2$. Denote $\sum_{j=2}^{n} q_{j}=q^{\prime}$. Then $\prod_{j=2}^{n} q_{j} ! \leqq e^{O(q)} q^{\prime} ! m^{-q}$. Given $m$, there are $\left(\begin{array}{c}n-1 \\ m\end{array}\right)$ choices of $\left\{q_{j_{k}}\right\}$, and $m \leqq q^{\prime} / 2$ since $q_{j_{k}} \geqq 2$. Also

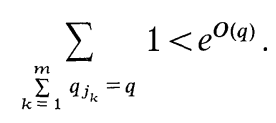

Hence

$$
A \leqq e^{O(q)} \sum_{q^{\prime}=0}^{q} \sum_{m=1}^{q^{\prime} / 2}\left(\begin{array}{c}
n-1 \\
m
\end{array}\right) q^{\prime} !\left(\frac{3 q}{2}-q^{\prime}\right) ! m^{-q^{\prime}} .
$$

Let first $n=\alpha q, \alpha \leqq 1 / 2$. Then $\left(\begin{array}{c}n-1 \\ m\end{array}\right)<2^{q / 2}$ and

$$
\begin{aligned}
A & \leqq e^{O(q)} \sum_{q^{\prime} m} \sum^{\prime} !\left(\frac{3 q}{2}-q^{\prime}\right) ! m^{-q^{\prime}} \leqq e^{O(q)} \frac{3 q}{2} ! \\
& \leqq e^{O(q)} n^{q / 2} \alpha^{-q / 2} q !
\end{aligned}
$$

If $n>\frac{q}{2}$, then we use $\left(\begin{array}{c}n-1 \\ m\end{array}\right)<n^{m}(m !)^{-1}$ and

$$
\sum_{m=1}^{q^{\prime} / 2}(m !)^{-1} m^{-q^{\prime}} n^{m-q^{\prime} / 2} \leqq e^{O\left(q^{\prime}\right)}\left(\frac{q^{\prime}}{2} !\right)^{-1}
$$


to derive

$$
\begin{aligned}
A & \leqq e^{O(q)} n^{q / 2} \sum_{q^{\prime}=0}^{q} q^{\frac{q^{\prime}-q}{2}} \frac{q^{\prime}}{2} !\left(\frac{3 q}{2}-q^{\prime}\right) ! \\
& \leqq e^{O(q)} n^{q / 2} q ! .
\end{aligned}
$$

(32)-(34) imply the Lemma.

\section{The "Dual" Representation}

In this section we bound the general remainder to the $1 / n$ expansion by making use of the dual representation. The idea is to generate the expansion and the remainder in the dual representation and then transform back to the $\phi$-representation, where the remainder is estimated.

To define the dual representation we first introduce the lattice approximation to the model, which converges to the continuum theory ([2], Chap. VII). For notational simplicity, let us assume the lattice spacing $\delta=1$. $i \in \Lambda$ will now denote the integral points of $\Lambda$. The partition function can thus be written as

$$
Z(\Lambda)=\frac{\int e^{V(\phi)} e^{-1 / 2(\phi,(-\Delta+1) \phi)} \prod_{i \in \Lambda} d^{n} \phi_{i}}{\int e^{-1 / 2(\phi,(-\Delta+1) \phi)} \prod_{i \in \Lambda} d^{n} \dot{\phi}_{i}}
$$

where

$$
V(\phi)=-\lambda(4 n)^{-1} \sum_{i \in \Lambda}\left(\left(\phi_{i}^{2}-n b\right)^{2}-4 b \phi_{i}^{2}+2 n b^{2}\right)
$$

and

$$
b=(-\Delta+1)_{i i}^{-1} \equiv C_{i i} .
$$

Writing

$$
\begin{aligned}
e^{-\frac{\lambda}{4 n}\left(\phi^{2}-n b\right)^{2}}= & \left(\frac{n}{\pi \lambda}\right)^{1 / 2} e^{-\frac{\lambda}{4 n}\left(\phi^{2}-n b\right)} \int e^{i a\left(\phi^{2}-n b\right)} \\
& \cdot e^{-\frac{n}{\lambda}\left(a+i \frac{\lambda b}{n}\right)^{2}} d a
\end{aligned}
$$

and carrying out the $\phi$ integrals we get

$$
Z(\Lambda)=\left(\frac{n}{\pi \lambda}\right)^{|\Lambda| / 2} \int d^{|\Lambda|} a \operatorname{det}(1-2 i C a)^{-n / 2} e^{-\frac{n}{\lambda} \operatorname{tr} a^{2}-i(n+2) b \operatorname{tr} a} .
$$

$1 / n$ expansion is thus a loop expansion about a saddle point.

After scaling $a \rightarrow z \lambda^{1 / 2} a, z \equiv n^{-1 / 2}$

$$
Z(\Lambda)=2^{|\Lambda|} \operatorname{det}\left(1+\lambda C^{(2)}\right)^{1 / 2} \int e^{g} d \gamma,
$$


where $d \gamma$ is the gaussian measure on $\mathbb{R}^{|\Lambda|}$ with covariance $\frac{1}{2}\left(1+\lambda C^{(2)}\right)^{-1}$ [recall that $\left.C_{i j}^{(2)}=\left(C_{i j}\right)^{2}\right]$ and

$$
g(a)=-i(n+2) z \lambda^{1 / 2} b \operatorname{tr} a-\frac{n}{2} \operatorname{tr} \log \left(1-2 i z \lambda^{1 / 2} C a\right)+\lambda \operatorname{tr}(C a)^{2} .
$$

The Taylor expansion of $g$ about $a=0$ is

$$
g(a) \sim-2 i b z \lambda^{1 / 2} \operatorname{tr} a+\frac{1}{2} \sum_{k=3}^{\infty} \frac{\left(2 i \lambda^{1 / 2}\right)^{k}}{k} z^{k-2} \operatorname{tr}(C a)^{k} .
$$

Wick ordering thus chooses the saddle point up to an $O(z)$ linear term. Note, that as $\delta \rightarrow 0 \quad b=C_{i i} \rightarrow \infty$; hence the first term in (37) serves to perform the necessary cancellations.

Fig. 4

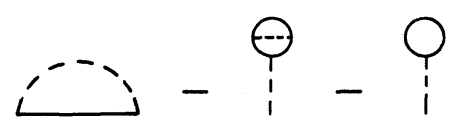

Let us see how these cancellations show up in the formal expansion. The first order graphs for the self energy are given in Fig. 4, where the dotted line is $K$. Each graph is divergent as $\delta \rightarrow 0$ since $K=1-\lambda L$ and thus $\sum_{j} C_{i j} K_{i j}$ involves $C_{i i}=b$. However, the sum of the divergent parts is

$$
b-b C^{(2)}\left(1+\lambda C^{(2)}\right)^{-1}-b\left(1+\lambda C^{(2)}\right)^{-1}=0 .
$$

In general the expansion coefficients involve graphs with lines $C$ and $L$ and vertices $C C C C$ and $C L C$ and tadpoles $C_{i i}$ are included. Divergences come from $C_{i i}$ and the $\delta$-parts of $K$ 's. Note, that not all $K$ 's in the graphs are divergent. In fact, let $G$ be a connected graph as above. Then we can prove

Proposition 10. If $G$ doesn't include as a part $C_{i j} K_{i j}$ or $C_{i i}$, then $\lim _{\delta \rightarrow 0} G$ exists.

Proof. If such terms are excluded, the claim is equivalent, after inserting $K=1-\lambda L$, to the assertion, that all connected graphs with lines and vertices as above, have a finite limit as $\delta \rightarrow 0$, if all tadpoles $C_{i i}$ are excluded. But these are just the graphs of a : $\phi^{4}$ : theory with some $\left(C_{i j}\right)^{2}$ parts replaced by $L_{i j}$. By Lemma 2 this replacement doesn't change the analytical structure.

It is also easy to cancel $b$ 's in the general order of $1 / n$ following the ideas of [1], Chap. 8. However, we have to cancel these divergences nonperturbatively in the remainder. Fortunately this can be done by generating the expansion in a suitable way, as will now be shown.

In order to generate the expansion in a-representation we start by making the dual transformation

$$
\begin{aligned}
\frac{d p}{d \lambda} & =\frac{\lambda}{4 n} \sum_{j} C_{i j}\left\langle: \phi_{i}^{(1)} \phi_{i}^{2}:: \phi_{j}^{(1)} \phi_{j}^{2}:\right\rangle_{\phi} \\
& =-\sum_{j} C_{i j}\left\langle\left(a_{i} a_{j}-\frac{1}{2} \delta_{i j}\right) H_{i j}\right\rangle,
\end{aligned}
$$


where $\langle\cdot\rangle$ is the expectation in the normalized measure $e^{g} d \gamma$ and $H=\left(-\Delta+1-2 i z \lambda^{1 / 2} a\right)^{-1}$. In the second step we used the fact that $\phi^{(\alpha)}\left(\phi^{2}-(n+2) b\right)=: \phi^{(\alpha)} \phi^{2}$ : together with the following transformation formula.

Lemma 11. Let I be a collection of points in $\Lambda$, the same point possibly occuring several times. Then

$$
\begin{aligned}
& \left\langle\prod_{i \in I} a_{i}\right\rangle=\left(\frac{i}{2}\right)^{|I|}\left(\frac{\lambda}{n}\right)^{|I| / 2} \\
& \cdot \sum_{\substack{J \subset I \\
|J| \text { even }}}-\left(\frac{2 n}{\lambda}\right)^{|J| / 2} \sum_{p \in P(J)}\left\langle\prod_{i \in I \backslash J} Q\left(\phi_{i}^{2}\right)\right\rangle \prod_{\left\langle j j^{\prime}\right\rangle \in p} \delta_{j j^{\prime}},
\end{aligned}
$$

where $P(J)$ denotes the set of pairings of $J$ and $Q(x)=x-(n+2)$.

Proof. Note that

$$
\begin{aligned}
& \pi^{-1 / 2} \int(i a)^{k} \exp \left[i a z \lambda^{1 / 2}\left(\phi^{2}-n b\right)-\frac{n}{\lambda}\left(z \lambda^{1 / 2} a+i \frac{\lambda b}{n}\right)^{2}-\frac{\lambda b}{n}\left(\phi^{2}-n b\right)\right] d a \\
& =(n / \lambda)^{k / 2}\left(\partial / \partial\left(\phi^{2}-n b\right)+\frac{\lambda b}{n}\right)^{k} \exp \left[-\frac{\lambda}{4 n}\left(\phi^{2}-n b\right)^{2}\right] \\
& \equiv(n / \lambda)^{k / 2} Q_{k}\left(\phi^{2}\right) \exp \left[-\frac{\lambda}{4 n}\left(\phi^{2}-n b\right)^{2}\right] .
\end{aligned}
$$

Since $Q_{k+1}=Q_{1} Q_{k}+\partial Q_{k}$ we get $\partial Q_{k}=k \beta Q_{k-1}\left(\beta=-\frac{\lambda}{2 n}\right)$ by induction:

$$
\begin{aligned}
\partial Q_{k+1} & =\beta Q+Q_{1} k \beta Q_{k-1}+k(k-1) \beta^{2} Q_{k-2} \\
& =\beta Q_{k}+k \beta\left(Q_{1} Q_{k-1}+(k-1) \beta Q_{k-2}\right)=(k+1) \beta Q_{k} .
\end{aligned}
$$

Again by induction it is easy to check that

$$
Q_{k}=\sum_{l=0}^{\left[\frac{k}{2}\right]} \frac{k !}{l !(k-2 l) !}\left(\frac{\beta}{2}\right)^{l}\left(Q_{1}\right)^{k-2 l} .
$$

If all $i \in I$ are identical, the claim follows since

$$
2^{l} \sum_{\substack{J \subset I \\
|J|=2 l}} \sum_{\substack{ \\
\mid P_{(}(J)}} 1=2^{l}\left(\begin{array}{c}
|I| \\
2 l
\end{array}\right) \frac{(2 l) !}{2^{l} l !}=\frac{|I| !}{l !(|I|-2 l) !} .
$$

Let $i_{1}=i_{2}=\ldots=i_{s}$ be different from other $i \in I$. We can then apply (39) to $\left(a_{i_{1}}\right)^{s}$ and induction to the other $a$ 's to prove the claim.

The expansion is generated using integration by parts. We get

$$
\begin{aligned}
& \left\langle a_{i} F\right\rangle=\frac{1}{2} \sum_{j} K_{i j}\left\langle\left(\partial_{j}+\partial_{j} g\right) F\right\rangle, \\
& \partial_{j} g=-2 i b \lambda^{1 / 2} z-4 i z \lambda^{3 / 2} \operatorname{tr} P_{j} C a H a C,
\end{aligned}
$$


where $P_{j}$ is the projection to the $j$ : th coordinate. We will also need

$$
\partial_{j} H=2 i z \lambda^{1 / 2} H P_{j} H \text {. }
$$

Let us now cancel the divergent $b$ 's. We proceed recursively. (38) is a special case of the expectation ( $F$ is in general a product of $a_{i}$ 's and $H_{j k}{ }^{\prime} \mathrm{s}$ )

$$
\left\langle\left(a_{i} a_{j}-\frac{1}{2} \delta_{i j}\right) H_{i j} F\right\rangle
$$

which by (40a) equals

$$
\begin{aligned}
& \left\langle\left(\frac{1}{2} K_{i j}-\frac{1}{2} \delta_{i j}\right) H_{i j} F+\frac{1}{2} a_{j} K_{i l}\left(\partial_{l}+\partial_{l} g\right) H_{i j} F\right\rangle \\
& \quad=-\lambda\left\langle L_{i j} H_{i j} F\right\rangle+\frac{1}{2}\left\langle a_{j} K_{i l}\left(\partial_{l}+\partial_{l} g\right) H_{i j} F\right\rangle
\end{aligned}
$$

(repeated indices are summed over except $i$ and $j$ ). Now we use (40b,c)

$$
\begin{aligned}
& \frac{1}{2} K_{i k}\left(\partial_{k}+\partial_{k} g\right) H_{i j} F=\frac{1}{2} K_{i k} H_{i j} \partial_{k} F \\
& \quad+\frac{1}{2} K_{i k}\left[2 i z \lambda^{1 / 2} H_{i k} H_{k j}+\left(-2 i b z \lambda^{1 / 2}-4 i z \lambda^{3 / 2} \cdot(\mathrm{CaHaC})_{k k}\right) H_{i j}\right] F .
\end{aligned}
$$

Writing now

$$
(\mathrm{CaHaC})_{k k}=\frac{1}{2} C_{k l} H_{l l} C_{l k}+C_{k l} H_{l m} C_{m k}\left(a_{l} a_{m}-\frac{1}{2} \delta_{l m}\right)
$$

and noting that

$$
\frac{1}{2} K_{i k}\left[-2 i z \lambda^{3 / 2} C_{k l} H_{l l} C_{l k}\right]=-i z \lambda^{1 / 2} H_{i i}+i z \lambda^{1 / 2} K_{i l} H_{l l}
$$

[since $\left.\left(K^{-1}\right)_{i j}=\delta_{i j}+\lambda\left(C_{i j}\right)^{2}\right]$ together with

$$
K_{i k} H_{i k} H_{k j}=H_{i i} H_{i j}-\lambda L_{i k} H_{i k} H_{k j}
$$

we get (recall that $b=C_{l l}$ )

$$
\begin{aligned}
& \frac{1}{2} K_{i k}\left(\partial_{k}+\partial_{k} g\right) H_{i j} F=-i z \lambda^{3 / 2} L_{i k} H_{i k} H_{k j} F \\
& \quad+i z \lambda^{1 / 2} K_{i l}\left(H_{l l}-C_{l l}\right) H_{i j} F+\frac{1}{2} K_{i k} H_{i j} \partial_{k} F \\
& \quad-2 i z \lambda^{3 / 2} K_{i k} C_{k l} H_{l m} C_{m k}\left(a_{l} a_{m}-\frac{1}{2} \delta_{l m}\right) H_{i j} F .
\end{aligned}
$$

Note that $H_{l l}-C_{l l}=2 i z \lambda^{1 / 2}(\mathrm{CaH})_{l l}$. Thus

$$
\begin{aligned}
& \left\langle\left(a_{i} a_{j}-\frac{1}{2} \delta_{i j}\right) H_{i j} F\right\rangle=-\lambda\left\langle L_{i j} H_{i j} F\right\rangle+\frac{1}{2}\left\langle K_{i k} a_{j} H_{i j} \partial_{k} F\right\rangle \\
& \quad-i z \lambda^{3 / 2}\left\langle L_{i k} a_{j} H_{i k} H_{k j} F\right\rangle-2 z^{2} \lambda\left\langle K_{i l}(C a H)_{l l} a_{j} H_{i j} F\right\rangle \\
& \quad-2 i z \lambda^{3 / 2}\left\langle\left(a_{l} a_{m}-\frac{1}{2} \delta_{l m}\right) H_{l m} F^{\prime}\right\rangle,
\end{aligned}
$$

where $F^{\prime}$ can be read off from $\left(41^{\prime}\right)$ and $(42)$.

We use (42) and (43) now recursively, starting with $F=1$ in (38). The last term of (43) is again of the form (41), with new $F$. In the other terms of (43) we first integrate the $a$ 's by parts and apply (42), getting again a term of the form (41) and

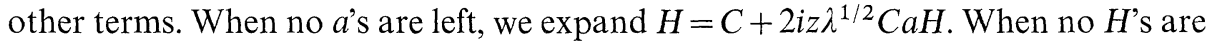
left, we have a term of the expansion. Note that in this way we cancelled all the $b$ 's and the $\delta$-parts of the troublesome $K$ 's (Proposition 10), since no $K_{i j} C_{i j}$ factors appear, only $L_{i j} C_{i j}$.

After expanding $K=1-\lambda L$, the expansion coefficients $p_{k}$ consist of connected graphs with lines and vertices as before and no tadpoles $C_{i i}$ appear. Since we 
always first integrate $a$ 's and only when none are left, expand $H$ 's, each $a_{j}$ in the remainder appears in the combination $a_{i} H_{i j}$. Moreover, there is only one $a_{i} H_{i j} a_{j}$, namely in the form $H_{i j}\left(a_{i} a_{j}-\frac{1}{2} \delta_{i j}\right)$. Thus the remainder is a sum of terms, which can be represented by graphs as above, with external "legs" as in Fig. 5 and some $C$ 's replaced by $H$ 's, such that the graphs remain connected when the $H$ 's are removed. [This can easily be seen from (42) and (43); e.g. $(\mathrm{CaH})_{l l}$ has $C_{k l} H_{k l}$. Recall that (38) includes a $C_{i j}$.]

Fig. 5

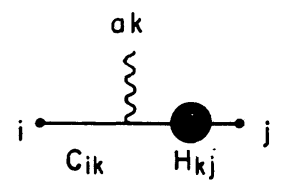

The terms in the remainder are thus of the form

$$
\begin{aligned}
T_{r_{1} r_{2}}= & \sum_{(i)(l)(s)(t)}\left(z \lambda^{3 / 2}\right)^{m_{1}}\left(z \lambda^{1 / 2}\right)^{m_{2}} G(C, \lambda L,(i), \ldots,(t)) \\
& \cdot\left\langle H_{i_{1} i_{2}}\left(a_{i_{1}} a_{i_{2}}-\frac{1}{2} \delta_{i_{1} i_{2}}\right) \prod_{\alpha=3}^{r_{1}} a_{i_{\alpha}} H_{i_{\alpha} l_{\alpha}} \prod_{\beta=1}^{r_{2}} H_{s_{\beta} t_{\beta}}\right\rangle,
\end{aligned}
$$

where $m_{1}+m_{2}=2 m$ for $R_{m}$. The factors of $i$ and 2 are included in $G$. We can now apply Lemma 11 to transform (44) to the $\phi$-representation. Since each expansion step which brings a factor of $z$ produces at most one new $H, r_{1}+r_{2} \leqq 2 m$. Let $n \geqq 2 m$. Then we can write

$$
\begin{aligned}
T_{r_{1} r_{2}}= & \sum_{(i) \ldots(t)}\left(z \lambda^{3 / 2}\right)^{m_{1}}\left(z \lambda^{1 / 2}\right)^{m_{2}} G(C, \lambda L,(i), \ldots,(t)) \\
& \cdot \sum_{J \subset I} \sum_{p \in P(J)}^{\prime}(-2)^{|J| / 2}\left(\frac{i}{2}\right)^{r_{1}}\left(\frac{\lambda}{n}\right)^{\left(r_{1}-|J| / 2\right)} \prod_{\left\langle i i^{\prime}\right\rangle \in p} \delta_{i i^{\prime}} \\
& \cdot\left\langle\prod_{i \in I \backslash J}: \phi_{i}^{(\alpha(i))} \phi_{i}^{2}: \prod_{j \in J} \phi_{j}^{(\beta(j))} \prod_{\alpha=3}^{r_{1}} \phi^{(\gamma(\alpha))} \prod_{\beta=1}^{r_{2}} \phi_{s_{\beta}}^{(\delta(\beta))} \phi_{t_{\beta}}^{(\delta(\beta))}\right\rangle,
\end{aligned}
$$

where each $c \in[1,2, \ldots, n]$ in $\phi^{(c)}$ appears only twice. We denoted $I=\bigcup_{\alpha=1}^{r_{1}} i_{\alpha}$ and as in Lemma 3, $|J|$ is even. $\sum_{p}^{\prime}$ means, that pairings $p$, for which $\left\langle i_{1} i_{2}\right\rangle \in p$, are excluded [because of the $-\frac{1}{2} \delta_{i_{1} i_{2}}$ term in (44)]. From (45) we see that all the $b$ 's are indeed cancelled in the remainder since only Wick powers occur.

Since (45) is in $\phi$-representation, we can take the continuum limit. The expectation in (45) is estimated as the first order term in Sect. 3. We first localize the kernels $S^{\alpha}$ smearing the (Wick) monomials $P_{\alpha}\left(\chi_{i}\right.$ is the characteristic function of $\Delta_{i}$ )

$$
P_{\alpha}\left(S^{\alpha}\right)=\sum_{i \in A \cap \mathbb{Z}^{2}} P_{\alpha}\left(\chi_{i} S^{\alpha}\right) \equiv \sum_{i} P_{\alpha}\left(S^{\alpha}(i)\right) .
$$

The $P_{\alpha}\left(S^{\alpha}\right)$ are $v_{i}\left(C_{y}\right),\left(\phi^{(j)} \phi^{(l)}\right)\left(K_{(y)}^{(2)}\right)(j \neq l)$ and $\phi^{(m)}\left(K_{(y)}^{(3)}\right)$ where

$$
K_{(y)}^{(2)}(x)=C\left(y_{1}-x\right) C\left(y_{2}-x\right) \quad \text { or } \quad L(y-x)
$$

and

$$
K_{(y)}^{(3)}(x)=\prod_{i=1}^{3} C\left(y_{i}-x\right) \quad \text { or } \quad L\left(y_{1}-x\right) C\left(y_{2}-x\right) .
$$


Thus we can write, absorbing constants to $G$ :

$$
\begin{aligned}
T_{r_{1} r_{2}}= & \sum_{J, p}(\lambda / n)^{\left(r_{1}-|J|\right) / 2} n^{-m} \lambda^{1 / 2\left(3 m_{1}+m_{2}\right)} \\
& \cdot \sum_{\left(i_{l}\right)} \int \prod_{l} d\left(y_{l}\right) G\left(\left(y_{l}\right), J\right)\left\langle\prod_{l} P_{\alpha(l)}\left(S_{\left(y_{l}\right)}^{\alpha(l)}\left(i_{l}\right)\right)\right\rangle .
\end{aligned}
$$

Let $\langle\cdot\rangle_{\Delta_{i}}$ be the gaussian measure with covariance $C_{\Delta_{i}}^{N}$ and let

$$
Q_{i}=\prod_{i_{l}=i} P_{\alpha(l)}\left(S_{\left(y_{l}\right)}^{\alpha(l)}\left(i_{l}\right)\right) \text {. }
$$

Then using chessboard estimates, Hölder's inequality and conditioning to $\bigoplus_{i \in A \cap \mathbb{Z}^{2}} C_{\Delta_{i}}^{N}$ as in Sect. 3 we can estimate $(1 / r+1 / q=1)$

$$
\left|\left\langle\prod_{l} P_{\alpha(l)}\left(S_{\left(y_{l}\right)}^{\alpha(l)}\left(i_{l}\right)\right)\right\rangle\right| \leqq \prod_{i}\left\langle Q_{i}^{q}\right\rangle_{\Delta_{i}}^{1 / q} e^{r^{-1} p(r \lambda)-p(\lambda)} .
$$

$\left\langle Q_{i}^{q}\right\rangle_{\Delta_{i}}$ is a gaussian integral, which is estimated as in Lemma 4.

Lemma 12. Let $t_{i}$ be the number of $v_{\alpha}$ factors in $Q_{i}$ and $d_{i}+3 t_{i}$ the total degree of $Q_{i}$. Then

$$
\left\langle Q_{i}^{q}\right\rangle^{1 / q} \leqq n^{t_{i} / 2} \Gamma\left(\frac{t_{i}}{2}\right)\left(\beta_{1} q^{1 / 2}\right)^{t_{2}+d_{l}} m_{i}^{t_{i} / 2} \prod_{i_{l}=i}\left\|S_{\left(y_{l}\right)}^{\alpha(l)}\right\|_{L^{p(\alpha)}\left(\Delta_{i}\right)},
$$

where $p(1)=4, p(2)=2, p(3)=4 / 3, \beta_{1}$ is a constant and $m_{i}=\max \left(1, \frac{q t_{i}}{n}\right)$.

Assuming Lemma 12 we now insert (47) to (46). The maximum power of $\lambda$ in (46) occurs when $m_{1}=2 m$. Since

$$
\sum t_{i}=r_{1}-|J| \text { and } \frac{1}{2}\left(r_{1}-|J|\right) \leqq r_{1} / 2 \leqq m+1,
$$

we get

$$
T_{r_{1} r_{2}} \leqq \lambda\left(\lambda^{4} / n\right)^{m} G(m, \lambda) H(m, q) e^{r-1} p(r \lambda)-p(\lambda),
$$

where

$$
G(m, \lambda)=\sup _{J \subset I, p \in P(J)} \sum_{\left(i_{l}\right)} \int_{l} d\left(y_{l}\right) G\left(\left(y_{l}\right), J\right) \prod_{l}\left\|S_{\left(y_{l}\right)}^{(\alpha(l))}\right\|_{L^{p(\alpha)}\left(\Delta_{t_{l}}\right)}
$$

and

$$
H(m, q)=\sum_{J, p} \sup _{\left(i_{l}\right)} \prod_{i} \Gamma\left(\frac{t_{i}}{2}\right)\left(\beta_{1} q^{1 / 2}\right)^{t_{2}+d_{i}} m_{i}^{t_{i} / 2} .
$$

We will later prove the following estimates for $G$ and $H$ :

Lemma 13. There exists a constant $\beta_{2}$ such that $G(m, \lambda) \leqq \beta_{2}^{m} \lambda^{2 m}$.

Lemma 14. Let $n>2 m$. Then there exists a constant $\beta_{3}$ such that

$$
H(m, q) \leqq \beta_{3}^{m} \Gamma\left(\frac{r_{1}}{2}\right) q^{4 m+3} .
$$


We can now prove Lemma 9 and Theorem 1. All we have to do is to estimate the number of $T_{r_{1} r_{2}}$ terms in $R_{m}$ since Lemmas 13 and 14 and (48) give us

$$
T_{r_{1} r_{2}} \leqq \lambda q^{3}\left(\lambda^{6} q^{4} n^{-1}\right)^{m} \beta_{4}^{m} \Gamma\left(\frac{r_{1}}{2}\right) e^{r^{-1} p(r \lambda)-p(\lambda)} .
$$

Let us denote terms in $R_{m}, m^{\prime}<m$, with $k$ 's and $l H$ 's by $t_{k, l}$. From (43) and the discussion following it we get for an elementary expansion step

$$
\begin{aligned}
t_{k, l} \rightarrow & {\left[\frac{1}{k+1} \sum_{m=0}^{k / 2} b_{k, m} t_{k+1-2 m, l+1}+\frac{l}{k-1} \sum_{m=0}^{\frac{k-2}{2}} b_{k-2, m}\right.} \\
& \left.\cdot t_{k-1-2 m, l}+(k-1) ! ! \sum_{m=0}^{l-1} t_{0, l-m}\right](k \text { even }), \\
t_{k, l} \rightarrow & {\left[\frac{1}{k+1} \sum_{m=0}^{(k+1) / 2} b_{k, m} t_{k+1-2 m, l+1}+\frac{l}{k-1} \sum_{m=0}^{\frac{k-1}{2}} b_{k-2, m} t_{k-1+2 m}\right] \text { (k odd), } }
\end{aligned}
$$

where

$$
b_{k, m}=(k+1)(k-1) \ldots(k+1-2 m) \text {. }
$$

Hence after $2 m$ such steps we get less than $(2 m) ! \beta_{5}^{m}$ terms of the form

$$
\left(\prod_{i=1}^{p} m_{i}\right) t_{r_{1}, l} \quad \text { where } 2 p+r_{1} \leqq 2 m, \quad m_{i}<2 m .
$$

Thus

$$
\prod_{i=1}^{p} m_{i} \Gamma\left(\frac{r_{1}}{2}\right) \leqq \beta_{6}^{m} m !
$$

and we get

$$
\left|R_{m}\right| \leqq(3 m) ! \lambda q^{3}\left(\lambda^{6} q^{4} n^{-1}\right)^{m} \beta_{7}^{m} e^{r^{-1} p(r \lambda)-p(\lambda)}
$$

Choosing $q=2$ in (52) we obtain Lemma 9 using (4).

For Theorem 1 we choose, as in the proof of Theorem $3, q=\alpha_{4} \lambda(\log \lambda)^{2}$ whence $\left|r^{-1} p(r \lambda)-p(\lambda)\right| \leqq O(1)$. Thus

$$
\left|R_{m}\right| \leqq(3 m) ! \lambda^{4}(\log \lambda)^{6}\left(\beta_{8} \lambda^{10}(\log \lambda)^{8} n^{-1}\right)^{m}
$$

which completes the proof of Theorem 1.

Remark. We note that for $m=1$ our previous result (Theorem 3 ) is better. This is because for general $m$ our method to generate the expansion is not very economical when the Hölder's inequality is used in the proof of Theorem 1. If we resolvent expanded $H$ 's before integrating the $a$ 's by parts, we would get fewer powers of $q$. However, the cancellations are harder to see and the expansion is not as systematic.

We close this chapter by proving Lemmas $12-14$. 
Proof of Lemma 12. As in Lemma 4 we expand each $: \phi^{2}:=\sum_{j=1}^{n}:\left(\phi^{(j)}\right)^{2}:$ and use the independence of $\phi^{(j)}$ 's and Wick's theorem. Since for each $j \phi^{(j)}$ factor occurs at most twice in $Q_{i}$ [excluding $:\left(\phi^{(j)}\right)^{2}$ : factors] the combinatorics is reduced to estimating

$$
A=\sum_{j=1} \prod_{j=q}^{n} \prod_{j=1}^{n}\left(\alpha_{j} \frac{q}{2}+q_{j}\right) !,
$$

where $\alpha_{j} \leqq 2, \sum \alpha_{j}=d_{i}$ and $q_{j} \neq i$.

We get

$$
A \leqq b_{0}^{q\left(t_{i}+d_{i}\right)} \prod_{j}\left(\alpha_{j} q / 2\right) ! \sum_{\Sigma q_{j}=t_{i} q} \prod q_{j} !
$$

We can now proceed as in Lemma 4 to bound the sum. The integrals of the kernels are estimated using Hölder's inequality repeatedly as before.

Proof of Lemma 13. We localize each $C$ and $L$ in $G$. Since each vertex connects an $L$ to $2 C$ 's or $4 C$ 's to each other we get by using Hölder's inequality repeatedly

$$
\begin{aligned}
& \sum_{\left(i_{l}\right)} \int \prod_{l} d\left(y_{l}\right) G\left(\left(y_{l}\right), J\right) \prod_{l}\left\|S_{\left(y_{l}\right)}^{\left(\alpha_{l}\right)}\right\|_{L^{p(\alpha)}\left(\Delta_{t_{l}}\right)} \\
& \quad \leqq \lambda^{B} b_{1}^{m} \sum_{\left(J_{\beta}\right)} \prod_{\langle\gamma \delta\rangle}\left\|G_{\gamma \delta}\right\|_{L^{p(\gamma \delta)}\left(\Delta_{j_{\gamma}} \times \Delta_{j_{\delta}}\right)},
\end{aligned}
$$

where $\left(j_{\beta}\right)$ runs through the localizations at each vertex and leg of $G,\langle\gamma \delta\rangle$ through the lines of the graphs, $j_{\gamma}$ and $j_{\delta}$ being the localizations at the endpoints and $G_{\gamma \delta}$ the corresponding kernel. $p(\gamma \delta)=4(2)$ if $G_{\gamma \delta}=C(L)$. B is the number of $L$ 's in $G$, thus $B \leqq 2 m$. Since

$$
\left\|G_{\gamma \delta}\right\|_{L^{p(\gamma \delta)}\left(\Delta_{i} \times \Delta_{j}\right)} \leqq b_{1} e^{-1 / 2|i-j|}
$$

and $G$ is connected,

$$
\sum_{\left(j_{\beta}\right)} \prod_{\langle\gamma \delta\rangle}\left\|G_{\gamma \delta}\right\| \leqq b_{2}^{m},
$$

and we get the claim.

Proof of Lemma 14. We note that

$$
\sum t_{i}=r_{1}-|J| \leqq r_{1} \leqq 2 m+2
$$

and from (45)

$$
\sum d_{i}=2\left(r_{1}+r_{2}-1\right) \leqq 4 m+2
$$

since each expansion step produces at most one new $H$ and so $r_{1}+r_{2}-2 \leqq 2 m$. Thus

$$
\prod \Gamma\left(\frac{t_{i}}{2}\right) \leqq \Gamma\left(\frac{r_{1}-|J|}{2}\right) \gamma_{1}^{m} .
$$


Since $\frac{1}{n} \sum t_{i} \leqq 1$ we can replace $m_{i}$ by $q$. Then

$$
\begin{aligned}
H(m, q) & \leqq q^{4 m+3} \gamma_{2}^{m} \sum_{J, p} \Gamma\left(\frac{r_{1}-|J|}{2}\right) \\
& =\gamma_{2}^{m} q^{4 m+3} \sum_{l=0}^{\left(r_{1} / 2\right)} r_{1} !\left(l !\left(r_{1}-2 l\right) !\right)^{-1} \Gamma\left(\frac{r_{1}-2 l}{2}\right) \\
& \leqq \Gamma\left(\frac{r_{1}}{2}\right) \beta_{3}^{m} q^{4 m+3} .
\end{aligned}
$$

Acknowledgements. I would like to thank Tom Spencer for numerous discussions, suggestions, and advice and Barry Simon for a careful reading of the manuscript.

\section{References}

1. Kupiainen, A.: Commun. Math. Phys. 73, 273 (1980)

2. Simon, B. : The $P(\phi)_{2}$ euclidean (quantum) field theory. Princeton: Princeton University Press 1974

3. Guerra, F., Rosen, L., Simon, B.: Ann. Math. 101, 111 (1975)

4. Guerra, F., Rosen, L., Simon, B.: Ann. Inst. H.Poincaré 25, 231 (1976)

5. Fröhlich, J., Simon, B.: Ann. Math. 105, 493 (1977)

6. Dimock, J., Glimm, J.: Adv. Math. 12 (1974)

7. Glimm, J., Jaffe, A.: Commun. Math. Phys. 44, 293 (1975)

8. D’Adda, A., Di Vecchia, P., Lusher, M.: Nucl. Phys. B152, 125 (1979)

Communicated by A. Jaffe

Received November 30, 1979 